\section{Agonizing switch in prostate cancer}

\section{By Amy Donner, Senior Editor}

Aragon Pharmaceuticals Inc. researchers have uncovered how a specific androgen receptor mutation results in turning second-generation antagonists for prostate cancer into agonists. ${ }^{1}$ The results could allow the company, which is being acquired by Johnson \& Johnson, to develop third-generation molecules that overcome the resistance mechanism.

Antiandrogen drugs were the first targeted therapy approved for prostate cancer and have substantially improved survival rates for patients. However, many castration-resistant prostate cancers (CRPCs) become resistant to antiandrogens because of elevated androgen receptor expression or mutation. ${ }^{2}$

Second-generation antiandrogens are effective against prostate cancers resistant to first-generation drugs because they antagonize both the overexpressed and the mutated receptors. These include marketed drug Xtandi enzalutamide, from Astellas Pharma Inc. and Medivation Inc., and Aragon's ARN-509, which is in Phase II testing.

Like with first-generation molecules, the duration of the therapeutic response to Xtandi or ARN-509 is limited because patients develop resistance to these compounds.

"With the selective pressure from therapeutics in cancer treatment, drug resistance seems inevitable. We need to determine whether we can anticipate and deal with drug resistance preemptively in the drug design and development process, instead of waiting to react," said Yang Shen, a research assistant professor at the Toyota Technological Institute at Chicago.

In a paper published in eLife earlier this year, Shen and collaborators at the Memorial Sloan-Kettering Cancer Center and The University of Chicago ran a prospective study to predict androgen receptor mutations that confer resistance to Xtandi and ARN-509. ${ }^{3}$

A mutagenesis-based screening approach in prostate cancer cell lines identified the F876L mutation as one that confers resistance to Xtandi and ARN-509. The mutation was validated in cell culture and xenograft models.

Now, an Aragon team led by James Joseph and Jeffrey Hager has gone a step further and showed the clinical relevance of the F876L mutation. In short, the mutation arises spontaneously in patients receiving ARN-
509 and turns the antagonistic effect of the drug into an agonistic one, thereby stripping the drug of its antitumor effect.

Joseph is a principal scientist at the company, and Hager is senior director of biology.

The group generated prostate cancer cell lines resistant to ARN-509 and Xtandi via prolonged in vitro selection in the presence of drug. DNA sequencing analyses showed that the F876L mutation emerged in three independent, resistant lines.

In the mutated cell lines, the drugs actually stimulated expression of androgen receptor-dependent genes and cell proliferation, suggesting the compounds agonized the mutant version of the androgen receptor. The conversion of an antagonistic to an agonistic effect by mutation was reminiscent of the effect of androgen receptor mutations acquired in response to treatment with first-generation antiandrogens. ${ }^{2}$

The group showed that the F876L mutation was responsible for reversing the response of prostate cancer cell lines to drugs in cell lines expressing the androgen receptor mutant and in castrated, xenograft male mouse models for prostate cancer.

The Aragon team then evaluated circulating tumor DNA isolated from plasma samples from patients enrolled in a Phase I trial of ARN509. The F876L mutation occurred in 3 of 29 ARN-509-treated patients. Two of those three patients had progressive disease.

Importantly, the mutation was not detected in pretreatment samples, which suggests the mutation is acquired during the course of treatment.

Results were published in Cancer Discovery.

According to Nigel Brooks, senior project director with AstraZeneca plc's Oncology Innovative Medicines Unit, "It is very important that the mutation has been identified independently in two separate laboratories, strengthening the notion that this is a true adaptive response to these particular antihormonal therapies."

\section{Third time is a charm}

According to Hager, the findings "set the stage for co-development of next-generation agents coupled with a blood-based companion diagnostic to guide treatment decisions."

Aragon would not comment on whether it is developing such agents or a diagnostic.

Brooks said AstraZeneca is trying to target the androgen receptor in a way that affects all mutations as well as full-length androgen receptor and splice variant forms of androgen receptor.

"The description of this specific mutation as well as other changes to the androgen receptor, such as alternate splicing, guides the development of further androgen receptor-targeted therapies that may overcome all these resistance mechanisms. The discovery of the mutation in human plasma DNA may provide a way to develop a blood-based diagnostic approach to guide treatment decisions," said Brooks.

"Once a full antagonist of F876L androgen receptor is in hand, 


\section{"The discovery of the mutation in human plasma DNA may provide a way to develop a blood-based diagnostic approach to guide treatment decisions." \\ -Nigel Brooks, AstraZeneca plc}

patients with rising PSA [prostate-specific antigen] levels or progressive disease should be screened while on second-generation antiandrogen therapy, and those acquiring the mutation should receive the nextgeneration therapy," said Hager.

Because of the small sample size in the clinical portion of the Cancer Discovery report, there is not yet a reliable estimate of the frequency of $\mathrm{F} 876 \mathrm{~L}$ in patients who develop progressive disease while taking second-generation antiandrogens.

"We need further information on the prevalence of this mutation and if it can be used as a predictive marker for response to other antiandrogen agents," said Brooks.

Aragon did not disclose whether determining the frequency of androgen receptor mutations is part of the Phase II trial design for ARN-509.

MSKCC is developing a third-generation antiandrogen. In the eLife paper, one of the center's compounds, dubbed DR103, inhibited growth of prostate cancer cell lines expressing the F876L mutant androgen receptor, whereas Xtandi or vehicle did not. MSKCC is testing the compound in mouse xenograft models for prostate cancer. ${ }^{4}$

MSKCC has filed patent applications covering DR103 and other chemical entities disclosed in its paper as well as the F876L mutation. Aragon also has filed a patent application on the F876L mutation. Medivation did not respond to requests for comment.

Donner, A. SciBX 6(25); doi:10.1038/scibx.2013.615

Published online June 27, 2013

\section{REFERENCES}

1. Joseph, J.D. et al. Cancer Discov.; published online June 18, 2013; doi:10.1158/2159-8290.CD-13-0226

Contact: Jeffrey H. Hager, Aragon Pharmaceuticals Inc., San Diego, Calif.

e-mail: jhager@aragonpharma.com

Contact: James D. Joseph, same affiliation as above e-mail: jjoseph@aragonpharm.com

2. Taplin, M.-E. Nat. Clin. Pract. Oncol. 4, 236-244 (2007)

3. Balbas, M.D. et al. eLife 2, e00499; published online April 9, 2013; doi:10.7554/eLife.00499

4. Lou, K.-J. SciBX 6(18); doi:10.1038/scibx.2013.429

\section{COMPANIES AND INSTITUTIONS MENTIONED}

Aragon Pharmaceuticals Inc., San Diego, Calif.

Astellas Pharma Inc. (Tokyo:4503), Tokyo, Japan AstraZeneca plc (LSE:AZN; NYSE:AZN), London, U.K. Johnson \& Johnson (NYSE:JNJ), New Brunswick, N.J. Medivation Inc. (NASDAQ:MDVN), San Francisco, Calif. Memorial Sloan-Kettering Cancer Center, New York, N.Y. Toyota Technological Institute at Chicago, Chicago, III. The University of Chicago, Chicago, III. 